\title{
Influence of magnetic anisotropy on the superferromagnetic ordering in nanocomposites
}

\author{
Mørup, Steen; Christiansen, Gunnar Dan
}

Published in:

Journal of Applied Physics

Link to article, DOI:

$10.1063 / 1.352446$

Publication date:

1993

Document Version

Publisher's PDF, also known as Version of record

Link back to DTU Orbit

Citation $(A P A)$ :

Mørup, S., \& Christiansen, G. D. (1993). Influence of magnetic anisotropy on the superferromagnetic ordering in nanocomposites. Journal of Applied Physics, 73(10), 6955-6957. https://doi.org/10.1063/1.352446

\section{General rights}

Copyright and moral rights for the publications made accessible in the public portal are retained by the authors and/or other copyright owners and it is a condition of accessing publications that users recognise and abide by the legal requirements associated with these rights.

- Users may download and print one copy of any publication from the public portal for the purpose of private study or research.

- You may not further distribute the material or use it for any profit-making activity or commercial gain

- You may freely distribute the URL identifying the publication in the public portal

If you believe that this document breaches copyright please contact us providing details, and we will remove access to the work immediately and investigate your claim 


\title{
Influence of magnetic anisotropy on the superferromagnetic ordering in nanocomposites
}

\author{
Steen Mørup and Gunnar Christiansen \\ Iaboratory of Applied Physics, Technical University of Denmark, DK-2800 Lyngby, Denmark
}

Magnetic interaction between ultrafine particles may result in superferromagnetism, i.e., ordering of the magnctic moments of particles which would be superparamagnetic if they were noninteracting. In this article we discuss the influence of the magnetic anisotropy on the temperature dependence of the order parameter.

Noninteracting ultrafine particles of magnetic materials are superparamagnetic at finite temperatures. However, if such particles are brought close together the magnetic interaction (dipole and/or exchange interaction) may result in an ordering of the magnetic moments below a critical temperature $T_{p}$. If the magnetic moments of the particles are parallel the ordering is called superferromagnetic. ${ }^{1-3}$ The order parameter $b(T)$ of a superferromagnetic system decreases with increasing temperature $T$ in a way which is similar to the behavior of normal ferromagnetic materials. ${ }^{1,3}$ Superferromagnetic materials may have interesting applications. In the vicinity of $T_{p}$ the initial susceptibility is very large. It has recently been suggested to use such materials for magnetic refrigeration. ${ }^{4}$

The magnetic energy of a particle $(i)$ in a superferromagnetic system may be written

$$
E_{i}=-K V(\mathbf{e} \cdot \mathbf{u})^{2}-\sum_{j} K_{m}^{i j} \mathbf{M}_{i} \cdot \mathbf{M}_{j}
$$

where $K$ is the magnetic anisotropy energy constant, $V$ is the volume, $\mathbf{e}$ is a unit vector in the easy magnetization direction, and $\mathbf{u}$ is a unit vector in the direction of the magnetization vector of the particle. $\mathbf{M}_{i}$ and $\mathbf{M}_{j}$ are the magnetization vectors of the particles $i$ and $j$, respectively, and the summation is taken over all neighboring particles $(j) . K_{m}^{i j}$ is a magnetic coupling constant for the interaction between the particles $i$ and $j$.

A simple mean-field theory has been used to calculate the magnetic properties of such a system. ${ }^{1-3}$ It is assumed that the summation over the neighbors $(j)$ in Eq. (1) can be replaced by an average over the neighboring particles:

$$
E_{i}=-K V(\mathbf{e} \cdot \mathbf{u})^{2}-K_{m} \mathbf{M}_{i} \cdot\langle\mathbf{M}\rangle .
$$

The $z$ direction is now defined as the direction of $\langle\mathbf{M}\rangle$ and the $\mathrm{x}$ direction is chosen such that it is in the plane defined by $\mathbf{e}$ and $\langle\mathbf{M}\rangle$. Using the spherical coordinates defined in Fig. 1, one finds that $\mathbf{e}=(\sin v, 0, \cos v)$ and $\mathbf{u}=(\sin \theta \cos \varphi, \sin \theta \sin \varphi, \cos \theta)$. We first assume that the easy directions of all the particles $j$ form the same angle $v$ with the $z$ direction but form different azimuthal angles. Thus when all azimuthal angles are equally represented in the summation in Eq. (1) the resulting field from the neighboring particles is directed along the $z$ direction.

Introducing the order parameter $b(T)=\langle M\rangle / M$, Eq. (2) can be written

$$
\begin{aligned}
E_{i}= & -K V(\sin v \sin \theta \cos \varphi+\cos v \cos \theta)^{2} \\
& -K_{m} M^{2} b(T) \cos \theta .
\end{aligned}
$$

For simplicity it is assumed in the following that the saturation magnetization of the particles is independent of temperature, i.e., that the temperature is well below the Curie temperature of the particles. It is easy to extend the calculations to the case where the magnetization of the particles is a function of temperature. ${ }^{1-3}$

The partition function for a particle with energy given by $\mathrm{Eq}$. (3) can be written

$$
Z=\frac{1}{4 \pi} \int_{0}^{2 \pi} d \varphi \int_{0}^{\pi} d \theta \sin \theta \exp \left(-E_{i} / k T\right)
$$

where $k$ is Boltzmann's constant.

The average magnetization of the particle $(i)$ along the $z$ direction is proportional to

$$
\begin{aligned}
\left\langle u_{z}\right\rangle & =b(T) \\
& =\frac{1}{4 \pi Z} \int_{0}^{2 \pi} d \varphi \int_{0}^{\pi} d \theta \sin \theta \cos \theta \exp \left(-E_{i} / k T\right) .
\end{aligned}
$$

In previous publications it has been shown that if the magnetic anisotropy is negligible $(K=0)$, Eq. (5) is reduced to

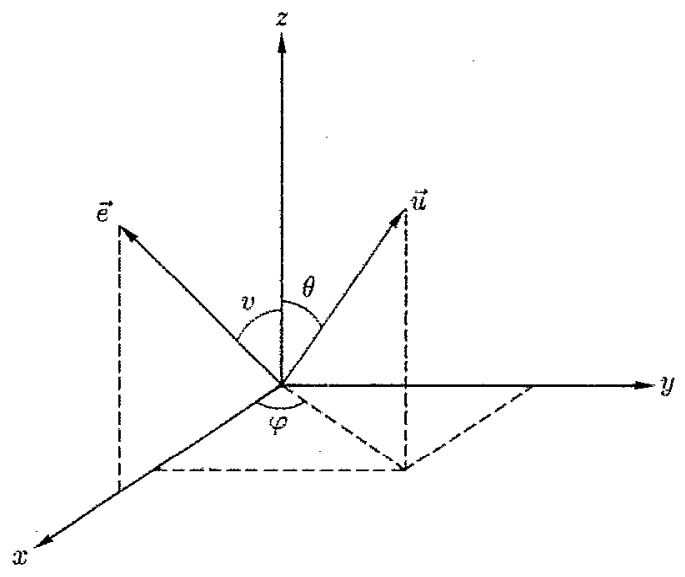

FIG. 1. Cartesian system and definition of the angles $\theta, \varphi$, and $v$ used in the calculations. 


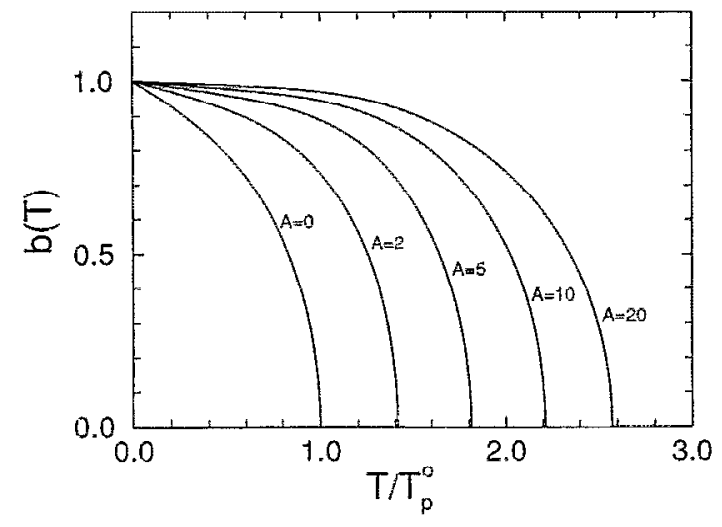

FIG. 2. Temperature dependence of the order parameter $b(T)$ for the indicated values of the anisotropy parameter $A$.

$$
b(T)=L\left(\frac{3 T_{p}^{0}}{T} b(T)\right),
$$

where $L(\quad)$ is the Langevin function and $T_{p}^{0}$ is the transition temperature given by

$$
T_{p}^{0}=\frac{K_{m} M^{2}}{3 k}
$$

Below the transition temperature the magnetic interaction between the particles results in ordering of the magnetic moments of the particles (superferromagnetic ordering). Above $T_{p}^{0}$ the particles are superparamagnetic $[b(T)$ $=0]$. This model has been shown to account for the measured temperature dependence of the average hyperfine field of ultrafine particles of goethite $(\alpha-\mathrm{FeOOH})$ (Refs. 1 and 3 ) and of imperfect $\mathrm{Fe} / \mathrm{Ag}$ multilayers. ${ }^{5}$

The magnetic anisotropy energy constant of ultrafine magnetic particles is often considerably larger than the bulk value. For small particles of metallic iron and iron oxides values of $K$ in the range $10^{4}-10^{6} \mathrm{~J} \mathrm{~m}^{-3}$ have been reported. For a particle with a diameter of about $8 \mathrm{~nm}$ and $K \approx 10^{5} \mathrm{~J} \mathrm{~m}^{-3}$ we find that $K V / k \simeq 2000 \mathrm{~K}$ which is much larger than the reported values of $T_{p}^{0}$. It is therefore surprising that the experimental results could be fitted well using a model in which $K=0$. In the following we present the results of some calculations of $b(T)$ for superferromagnetic systems with finite values of the magnetic anisotropy.

First we consider the case when $v=0$, i.e., the easy direction of magnetization is parallel to the mean-field direction. We introducc the anisotropy parameter $A=K V /$ $k T_{p}^{0}$. Figure 2 shows the calculated temperature dependence of $b(T)$ for various values of $A$. It is seen that the transition temperature $T_{p}$ increases with increasing values of $A$ and the shape of the curves also depends on the value of $A$. In Fig. 3 we have shown $T_{p} / T_{p}^{0}$ as a function of $A$. For $A \rightarrow \infty$ one finds that $T_{p} / T_{p}^{0} \rightarrow 3$ (corresponding to an Ising model for the superferromagnetic particles).

In Fig. 4 is shown the influence of the angle $v$ on $b(T)$ for $A=1.0$. It is found that $T_{p}>T_{p}^{0}$ for small values of $v$,

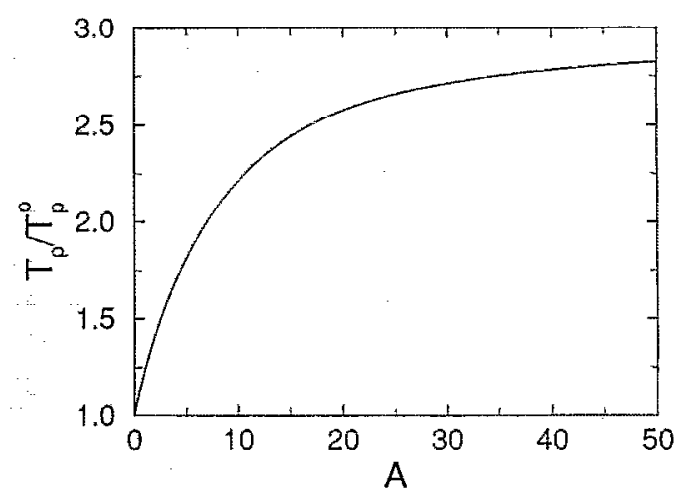

FIG. 3. Calculated dependence of the ordering temperature as a function of the anisotropy parameter $A$ in the case when $v=0$.

but $T_{p}<T_{p}^{0}$ for large values of the angle $v$. In a previous publication $^{6}$ it was shown that for small values of the anisotropy the value of $T_{p}$ is given by

$$
T_{p} \simeq \frac{K_{m} M^{2}}{3 k}+\frac{2}{15} \frac{K V}{k}\left(3 \cos ^{2} v-1\right) \text {. }
$$

In practice it may be expected that the easy directions of magnetization in a sample of ultrafine particles are randomly oriented. In this case the temperature dependence of the order parameter can be found by integrating over all orientations:

$b(T)=\frac{1}{2} \int_{0}^{\pi} d v \sin v \frac{\int_{0}^{\pi} d \theta \sin \theta \cos \theta \exp \left(-E_{i} / k T\right)}{\int_{0}^{\pi} d \theta \sin \theta \exp \left(-E_{i} / k T\right)}$,

where $E_{i}$ is given by Eq. (3).

Solving this equation for $b(T)$ numerically we obtain the results given in Fig. 5 for $A=0,1$, and 5. [The curve for $A=0$ is identical to that obtained by solving $\mathrm{Eq}$. (6).] In order to compare the shapes of the curves we have scaled the curves for $A=1$ and 5 such that the transition temperatures are equal to that for $A=0$. It is seen that both for

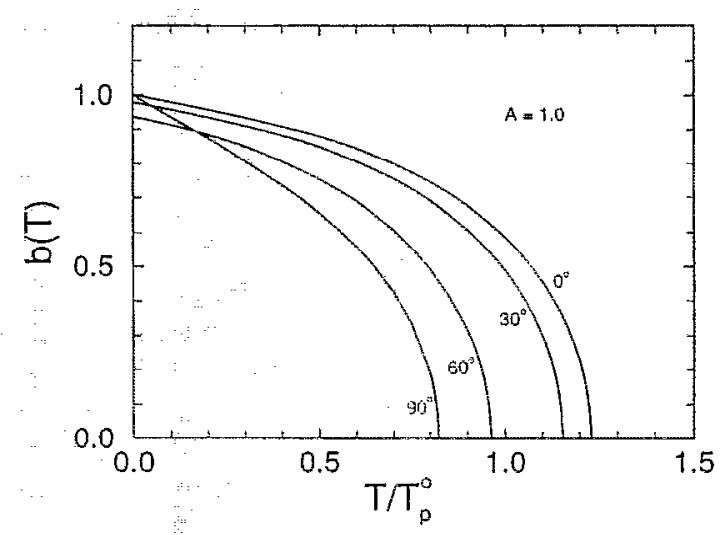

FIG. 4. Temperature dependence of the order parameter $b(T)$ for various angles $v$. The results were obtained for $A=1.0$. 


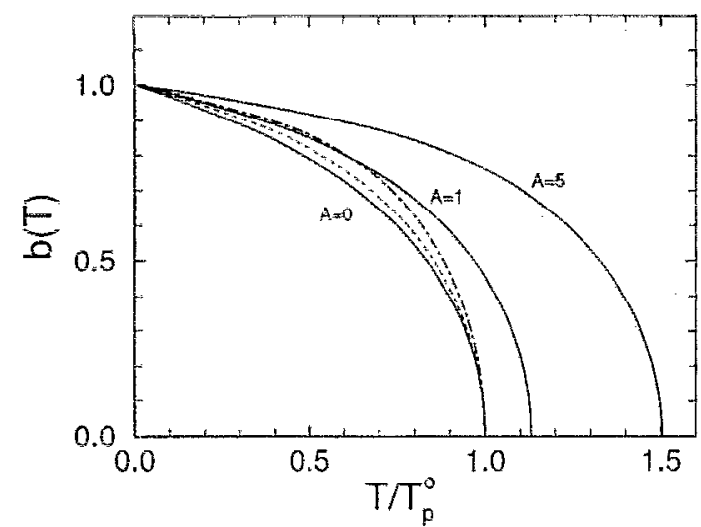

FIG. 5. Temperature dependence of the order parameter for a randum orientation of the easy directions. Results are shown for $A=0,1$, and 5 . The dotted and the dash-dotted lines are the curves for $A=1$ and 5 , respectively scaled to the same transition temperature as for $A=0$.
$A=1$ and 5 the scaled curves lie above that for $A=0$. However, the deviation is much less than that found for a fixed angle $v$, e.g., $v=0$. Thus it is found that a random orientation of the easy directions results in a temperature dependence of the order parameter which is close to that found for $A=0$. This may explain why the experimental results can be fitted well with a model with $A=0$.

The work was supported by the Danish Council for Technical Research.

'S. Mørup, M. B. Madsen, J. Franck, J, Villadsen, and C. J. W. Koch, J. Magn. Magn. Mater. 40, 163 (1983).

${ }^{2}$ S. Morup, P. H. Christensen, and B. S. Clausen, J. Magn. Magn. Mater. 68, $160(1987)$.

${ }^{3}$ S. Morup, Hyp. Int. 60, 959 (1990).

${ }^{4}$ L. H. Bennett, R. D. McMichael, L. J. Swartzendruber, R. D. Shull, and R. E. Watson, J. Magn. Magn. Mater. 104-107, 1094 (1992).

${ }^{5}$ S. Morup, G. Christiansen, and N. C. Koon, J. Magn. Magn. Mater. 104-107, 1793 (1992).

${ }^{\circ} \mathrm{S}$. Morup, in Studies of Magnetic Properties of Fine Particles and their Relevance to Materials Science, edited by J. L. Dormann and D. Fiorani (Elsevier, Amsterdam, 1992), p. 125. 